\title{
Potentiality of Binucleate Rhizoctonia Isolates as Root Rot Causing Pathogens on Faba Bean Maha H. Mohamed
}

Plant Pathol. Dept., Fac. Agric., Ain Shams Univ., Egypt.

\begin{abstract}
The present study was conducted to identify and determine the pathogenic capabilities of 78 binucleate Rhizoctonia isolates isolated from faba bean plants showing root rot and stem canker symptoms. Identification, depending on morphological features, has proved that isolates represented as: two isolates Rhizoctonia calle, 26 isolates $R$. fumigata, 48 isolates $R$. cerealis and two species are still unidentified due to edict of identification key. In vitro pathogenicity test was achieved for all binucleate isolates comparing with one accurate identified polynucleate Rhizoctonia solani (AG4-HGI). All isolates were found to be pathogenic and most of them showed the same degree of pathogenicity as $R$. solani. Potentiality of the most aggressive 4 isolates (belonging to $R$. cerealis) on faba bean as the causal of root rot was assessed comparing with polynucleate $R$. solani on disease incidence and on plant growth characters under greenhouse conditions. Binucleate tested isolates showed significantly greater disease incidence, and showed significant depressed effect on both shoots and roots compared with control. Results indicated the importance of binucleate Rhizoctonia spp. as causal agents of faba bean root rot.
\end{abstract}

Keywords: Binucleat Rhizoctonia spp., Morphological characters, Pathogenicity and Vicia faba L.

Rhizoctonia diseases cause significant losses of various important economic crops under greenhouse and field conditions (Botha et al., 2003; Sharma-Poudyal et al., 2015; Yang et al., 2015). Strains of polynucleate and other binucleate Rhizoctonia spp. are capable of attacking a wide range of plant hosts, causing severe symptoms including: seed decays, damping-off of seedlings, root rots, stem cankers, and foliage diseases (Botha et al., 2003; Simonetta et al., 2007; Sharma-Poudyal et al., 2015).

The genus Rhizoctonia includes a complex of genetically distinguishing species, with a wide virulence exhibit preference for certain hosts (Anderson, 1982). Both polynucleate and binucleate Rhizoctonia spp. have been divided into number of anastomosis groups (AGs) based on hyphal anastomosis (Ogoshi et al., 1983, Sneh et al., 1991). Rhizoctonia solani Kühn [teleomorph: Thanatephorus cucumeris (Frank) Donk] is a polynucleate species that has been divided to 14 AGs. Rhizoctonia zeae and $R$. oryzae are polynucleate with the holomorphs Waitea circinata var. zeae and W. cercinata var. circinata, respectively (Sneh et al., 1991). Binucleate Rhizoctonia spp. (holomorph: Ceratobasidium spp. and Tulasnella spp.) are divided into 19 AGs (Ogoshi et al., 1983; Ogoshi, 1985). 
Binucleate Rhizoctonia spp. and hypovirulent $R$. solani isolates have been demonstrated as a biocontrol agent against $R$. solani or Pythium spp. diseases in a variety of hosts (Cubeta and Echandi, 1991; Herr, 1995; Poromarto et al., 1998; Erper et al., 2013). As well, many researchers showed that binucleate Rhizoctonia spp. show ability as virulent, less virulent or avirulent on plant hosts (Eken and Demirci, 2003; 2004; Cedeno et al., 2006; Tuncer and Eken, 2013; Ünal et al., 2014; Sharma-Poudyal et al., 2015). Binucleate Rhizoctonia include several important plant pathogenic fungi on several host plants such as strawberries (Botha et al., 2003), potato (Araki et al., 1979), turfgrass (Burpee, 1980), peanut (Oniki and Araki, 1981; 1982), alfalfa (Cedeno et al., 2006), and ornamental plants (Hyakumachi et al., 2005; Molaei et al., 2014).

This study was undertaken to 1) Identify binucleate isolates of Rhizoctonia spp. isolated from faba bean plants showed root rot and stem canker. 2) Determine the pathogenic ability of isolated binucleate Rhizoctonia under laboratory and greenhouse condition. 3) Evaluate the effect of pathogenic isolates on growth characters of faba bean plants grown in infested soil.

\section{Materials and Methods}

\section{Isolation and identification of the pathogen:}

Faba bean plants (Vicia faba L.) with root rot and stem canker symptoms were collected from Qalyubia and Sharqia growing area. Root and basal stem portions with distinct lesions were rinsed with tap water to remove adhesive soil then cut into pieces and 3 parts were put in plates containing sterilized $2 \%$ water agar (WA). Plates were incubated at $25^{\circ} \mathrm{C}$ and examined after $24 \mathrm{hr}$. Rhizoctonia-Like mycelia were purified by hyphal-tip then sub-cultured on potato sucrose agar (PSA) media. Hyphal characteristics were examined microscopically to confirm that they matched with the description of Rhizoctonia spp. (Parmeter and Whitney, 1970).

Morphological features of mycelial growth were observed from upper side and down side of plates and all isolates were divided into two main groups: binucleate and polynucleate according to nuclei stain by trypan blue $(0.5 \%)$ dissolved in lactophenol (Burpee et al., 1978). Slide culture technique was used to determine number of nuclei in apical compartments. Sterilized glass slide was covered by PSA media and after solidification a small pit of fungal growth was put in the central part of the slide. Slides were put in sterilized petri dishes then incubated at $25 \pm 1^{\circ} \mathrm{C}$. The stain was added directly on young hyphae growing on agar media and left till hyphae were stained. Stained hyphae were covered with a microscopic slide cover and examined by light microscopy. Nuclei are stained dark blue. Seventy eight isolates represent as binucleate Rhizoctonia were identified depending on color of colonies, diameter of hyphae, measures of moniloid cells, and sclerotia characters according to Sneh et al. (1991). All parameters (hyphae and moniloid cells) were determined in 15 cells by using light microscopy at x 400 magnification. 


\section{Pathogenicity test:}

\subsection{Under laboratory conditions:}

Faba bean seeds (Giza $2 \mathrm{cv}$.) were rinsed with tap water, and then sterilized superficially with sodium hypochlorite $(2 \%)$ for 5 min. Seeds were washed with sterile distilled water then dried throw filter papers. For germination, seeds were placed in plastic box contained wetted filter paper in the dark. Apparently healthy germinated seeds have been selected then peeled.

All binucleate isolates were tested for their pathogenicity. Rhizoctonia solani AG4-HGI was isolated from faba bean and identified according to sequences of ITS1-5.8S rDNA-ITS4 (Mohamed et al., 2015a) and was used for comparison. Sterile germinated faba bean peeled seeds were put on fungal growth growing on potato sucrose agar medium. Plates were incubated at $25 \pm 1^{\circ} \mathrm{C}$ for three days in the dark, and then disease index was determined according to Mohamed et al. (2014) as follow: 1: apparently healthy seeds; 2 : very weak infection; 3 : moderate infection; 4 : very severe infection; 5 : seeds were died and completely covered by fungal mats. Three dishes (each contains 5 seeds) were used as replicates.

\subsection{Under greenhouse conditions:}

Four binucleate Rhizoctonia isolates Nos.1, 34, $38 \& 70$ which represent the aggressive isolates in vitro assay were used to determine their pathogenicity and their effects on morphological features of faba bean plants under greenhouse conditions.

\subsubsection{Preparation of fungal inoculum:}

Fungal inoculum was prepared on sand amended with Czapek Dox broth medium according to the method described by (Mohamed et al., 2014). Sand used in this study was washed with hydrochloric acid $(2 \mathrm{~N})$ then with distilled water and dried, finally distributed in $9 \mathrm{~cm}$ diameter petri dishes $(120 \mathrm{~g}$ sand/plate). Sand was drenched by Czapek Dox broth $\left(25 \mathrm{ml} /\right.$ plate). Dishes were autoclaved at $121^{\circ} \mathrm{C}$ for $30 \mathrm{~min}$. After cooling, dishes were infested by pieces of actively binucleate Rhizoctonia isolates growth or polynucleate $R$. solani isolate. Plates were incubated for 10 days in the dark at $25 \pm 1^{\circ} \mathrm{C}$. Sand dishes contained fungal growth were used as inoculum for infestation of sand pots. One dish of inoculum was used for each pot.

\subsubsection{Infestation of sand culture and cultivation of seeds:}

Washed and autoclaved sand was distributed in plastic pots diameter $10 \mathrm{~cm}$ by adding approximately $430 \mathrm{~g}$ sand/pot. Each pot was infested with fungal inoculum (one plate/pot). Infested pots were left for a week with a follow-up irrigation. Apparently healthy faba bean germinated seeds were sown in sand culture (3 seeds/pot) infested or not with binucleate isolates (Nos.1, 34, $38 \& 70$ ) and by one strain of polynucleate Rhizoctonia solani (AG4-HGI) as a comparison. Pots were irrigated when needed by sterilized distilled water and with Hogland's solution every two days. Each treatment consisted of ten replicates. 
2.2.3. Determination of disease index and plant growth characters under plastic house conditions:

Number of emerged plants was recorded. Plants were taken then roots were washed to remove adhered sand. Plant height $(\mathrm{cm})$, leaf number/ plant, foliage weight $(\mathrm{g})$ and root weight $(\mathrm{g})$ were determined 30 days after sowing. Aside from, disease index was determined according to Mohamed et al. (2015b) by using scale with a score range of 1 to 9 as follow: 1: no visible symptoms; 2: light discoloration with or without necrotic lesions (5\% of root tissue covered with lesions); $3: 15 \%$ of root tissue covered with lesions; $4: 25 \%$ of root tissue covered with lesions, the tissues remain firm with some deterioration of the root system; 5: 50\% of root tissue covered with lesions combined with rotting and reduction of root system; 6: 75\% of root tissues affected and combined with severe reduction in the root system; 7: root completely rotted after formation of the root system; 8: hypocotyls and root completely rotted after beginning of root formation; 9: rotted germinated seed and no root system was formed

\section{Statistical analyses:}

Standard deviation (SD) for all obtained means was calculated between data according to Ghahramani (2000).

\section{R e s u l t s}

\section{Isolation and identification of Rhizoctonia isolates:}

A total of 104 fungal isolates were isolated from faba bean roots with typical symptoms of Rhizoctonia root rot. Microscopic examination of nuclei stained revealed the presence of 26 isolates belong to polynucleate Rhizoctonia spp. and 78 isolates to binucleate Rhizoctonia spp. (Table, 1). As shown from Table 1, number of Rhizoctonia isolates isolated from plants collected from Qalyubia governorate was higher than that isolated from Sharqia governorate.

Binucleate Rhizoctonia isolates (78 isolates) have been selected to identify and procedure the pathogenicity test. Identification of 78 binucleate Rhizoctonia isolates according to morphology of colony on PDA media (according to Sneh et al., 1991), width of the main runner hyphae, and diameter of moniloid cells, showed that all isolates belong to three species: Rhizoctonia calle ( 2 isolates), $R$. cerealis (48 isolates), R. fumigatea (26 isolates) and two isolates are still unidentified (Tables 2 and 3).

Table 1. Nuclei number of Rhizoctonia spp. isolated from faba bean showing root rot symptoms

\begin{tabular}{|c|c|c|c|}
\hline Source of isolates & Number of Isolates & Polynucleate & Binucleate \\
\hline Qalyubia & 87 & 17 & 70 \\
\hline Sharqia & 17 & 9 & 8 \\
\hline Total & 104 & 26 & 78 \\
\hline$\%$ & & 25 & 75 \\
\hline
\end{tabular}


Table 2. Identification of $\mathbf{7 8}$ isolates of binucleate Rhizoctonia according to Sneh et al. (1991)

\begin{tabular}{|c|c|c|c|c|c|c|c|}
\hline 产 & $\frac{\frac{\dot{0}}{8}}{\frac{\pi}{8}}$ & 氞 & $\frac{\frac{3}{8}}{\frac{\pi}{8}}$ & 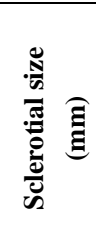 & 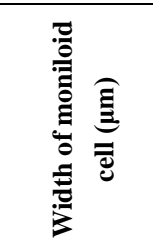 & 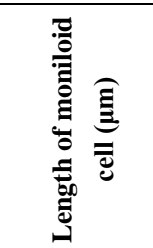 & 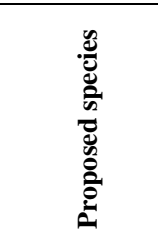 \\
\hline 1 & $\mathrm{~B}$ & $5.37 \pm 1.54$ & $\mathrm{~B}$ & $0.5-3.0$ & $7.10 \pm 1.54$ & $18.37 \pm 5.15$ & R. cerealis 1 \\
\hline 2 & $\mathrm{~B}$ & $4.16 \pm 1.64$ & $\mathrm{~B}$ & $0.5-3.0$ & $11.26 \pm 2.72$ & $23.22 \pm 5.15$ & R. cerealis 2 \\
\hline 3 & WY & $6.41 \pm 1.34$ & $\mathrm{~W}$ & $0.5-1.0$ & $7.10 \pm 2.29$ & $21.84 \pm 7.27$ & R. cerealis 3 \\
\hline 4 & WY & $5.89 \pm 1.54$ & $\mathrm{~W}$ & $0.5-1.0$ & $9.53 \pm 2.12$ & $19.76 \pm 4.26$ & R. cerealis 4 \\
\hline 5 & WY & $6.41 \pm 1.34$ & $\mathrm{~W}$ & $0.5-1.0$ & $7.32 \pm 2.27$ & $23.40 \pm 8.05$ & R. cerealis 5 \\
\hline $6 *$ & WY & $4.68 \pm 1.07$ & LB & $0.5-1.0$ & $5.54 \pm 0.91$ & $23.57 \pm 4.22$ & $R$. species 1 \\
\hline $7 *$ & WY & $3.98 \pm 2.38$ & LB & $0.5-1.0$ & $7.10 \pm 1.19$ & $22.01 \pm 3.38$ & R. cerealis6 \\
\hline $8 *$ & WY & $5.02 \pm 1.54$ & LB & $0.5-1.0$ & $7.97 \pm 1.82$ & $23.22 \pm 5.85$ & R. cerealis 7 \\
\hline $9 *$ & WY & $6.24 \pm 1.31$ & LB & $0.5-1.0$ & $8.49 \pm 1.54$ & $22.53 \pm 6.56$ & R. cerealis8 \\
\hline $10 *$ & WY & $6.06 \pm 1.26$ & LB & $0.5-1.0$ & $8.84 \pm 1.64$ & $18.89 \pm 5.33$ & R. fumigata 1 \\
\hline $11 *$ & WY & $6.06 \pm 1.26$ & LB & $0.5-1.0$ & $8.84 \pm 2.15$ & $19.06 \pm 4.67$ & R. fumigata 2 \\
\hline $12 *$ & WY & $5.20 \pm 0.98$ & LB & $0.5-1.0$ & $7.28 \pm 2.24$ & $19.93 \pm 2.53$ & R. fumigata3 \\
\hline $13 *$ & WY & $4.68 \pm 2.63$ & LB & $0.5-1.0$ & $5.02 \pm 0.67$ & $26.34 \pm 3.91$ & $R$. species 2 \\
\hline 14 & WY & $3.29 \pm 1.19$ & $\mathrm{~B}$ & $0.5-1.0$ & $12.48 \pm 2.24$ & $21.66 \pm 5.26$ & R. calle 1 \\
\hline 15 & WY & $3.98 \pm 1.66$ & $\mathrm{~B}$ & $0.5-1.0$ & $11.10 \pm 2.68$ & $20.62 \pm 4.65$ & R. calle 2 \\
\hline 16 & WY & $3.98 \pm 1.66$ & $\mathrm{~B}$ & $0.5-1.0$ & $10.40 \pm 3.25$ & $23.05 \pm 5.71$ & R. cerealis 9 \\
\hline 17 & WY & $3.81 \pm 1.34$ & $\mathrm{~B}$ & $0.5-1.0$ & $9.70 \pm 2.29$ & $22.36 \pm 4.89$ & R. cerealis 10 \\
\hline 18 & WY & $6.24 \pm 1.31$ & $\mathrm{~B}$ & $0.5-3.0$ & $9.53 \pm 2.53$ & $22.70 \pm 5.68$ & R. cerealis 11 \\
\hline 19 & WY & $6.58 \pm 1.66$ & $\mathrm{~B}$ & $2.0-7.0$ & $7.10 \pm 2.07$ & $17.16 \pm 4.48$ & R. fumigata 4 \\
\hline 20 & WY & $6.41 \pm 1.34$ & $\mathrm{~B}$ & $0.5-1.0$ & $9.18 \pm 2.92$ & $19.58 \pm 3.91$ & R. fumigata 5 \\
\hline 21 & WY & $6.76 \pm 1.31$ & A & ---- & $7.97 \pm 2.85$ & $19.93 \pm 4.46$ & R. fumigata6 \\
\hline 22 & WY & $6.06 \pm 1.60$ & $\mathrm{~B}$ & $0.5-1.0$ & $8.32 \pm 2.24$ & $21.66 \pm 5.53$ & R. cerealis 12 \\
\hline 23 & WY & $6.24 \pm 1.31$ & $\mathrm{~B}$ & $0.5-3.0$ & $8.14 \pm 3.23$ & $18.89 \pm 4.55$ & R. cerealis 13 \\
\hline 24 & WY & $6.76 \pm 1.31$ & $\mathrm{~B}$ & $0.5-3.0$ & $11.96 \pm 2.74$ & $24.44 \pm 5.54$ & R. cerealis 14 \\
\hline 25 & WY & $3.98 \pm 1.34$ & $\mathrm{~B}$ & $0.5-1.0$ & $8.46 \pm 2.08$ & $18.37 \pm 5.68$ & R. cerealis 15 \\
\hline 26 & $\mathrm{~B}$ & $6.58 \pm 1.34$ & $\mathrm{~B}$ & $0.5-3.0$ & $8.66 \pm 2.89$ & $18.37 \pm 6.40$ & R. cerealis 16 \\
\hline 27 & $\mathrm{~B}$ & $3.98 \pm 1.66$ & $\mathrm{~B}$ & $0.5-3.0$ & $10.40 \pm 4.50$ & $19.76 \pm 5.08$ & R. cerealis 17 \\
\hline 28 & $\mathrm{~B}$ & $6.58 \pm 1.34$ & $\mathrm{~B}$ & $0.5-3.0$ & $7.45 \pm 2.57$ & $15.94 \pm 5.80$ & R. cerealis 18 \\
\hline 29 & $\mathrm{~B}$ & $7.10 \pm 1.19$ & $\mathrm{~B}$ & $0.5-3.0$ & $8.32 \pm 1.75$ & $15.08 \pm 4.30$ & R. cerealis 19 \\
\hline 30 & B & $6.76 \pm 1.31$ & $\mathrm{~B}$ & $0.5-2.0$ & $7.10 \pm 2.68$ & $21.14 \pm 8.50$ & R. cerealis 20 \\
\hline 31 & $\mathrm{~B}$ & $6.68 \pm 1.33$ & $\mathrm{~A}$ & ---- & $9.47 \pm 3.16$ & $22.84 \pm 7.29$ & R. cerealis 21 \\
\hline 32 & $\mathrm{~B}$ & $6.24 \pm 1.64$ & $\mathrm{~B}$ & $0.5-3.0$ & $7.80 \pm 2.19$ & $22.01 \pm 5.63$ & R. cerealis 22 \\
\hline 33 & $\mathrm{~B}$ & $6.58 \pm 1.34$ & A & ---- & $8.66 \pm 2.12$ & $20.45 \pm 5.63$ & R. cerealis 23 \\
\hline 34 & $\mathrm{~B}$ & $6.58 \pm 1.34$ & $\mathrm{~B}$ & $0.5-3.0$ & $9.01 \pm 2.38$ & $19.93 \pm 4.88$ & R. cerealis 24 \\
\hline 35 & B & $6.58 \pm 1.66$ & $\mathrm{~B}$ & $0.5-3.0$ & $9.36 \pm 2.56$ & $19.76 \pm 5.36$ & R. cerealis 25 \\
\hline 36 & $\mathrm{~B}$ & $6.24 \pm 1.31$ & $\mathrm{~B}$ & $0.5-3.0$ & $8.32 \pm 3.83$ & $18.72 \pm 8.17$ & R. cerealis 26 \\
\hline 37 & $\mathrm{~B}$ & $6.06 \pm 1.88$ & $\mathrm{~B}$ & $0.5-3.0$ & $7.62 \pm 2.49$ & $15.94 \pm 3.78$ & R. cerealis 27 \\
\hline
\end{tabular}

Table 2.... continued 


\begin{tabular}{|c|c|c|c|c|c|c|c|}
\hline 38 & B & $6.76 \pm 1.64$ & $\mathrm{~B}$ & $0.5-3.0$ & $9.53 \pm 3.20$ & $22.18 \pm 5.37$ & R. cerealis 28 \\
\hline 39 & $\mathrm{~B}$ & $5.20 \pm 2.19$ & B & $0.5-3.0$ & $9.53 \pm 2.12$ & $16.46 \pm 5.44$ & R. cerealis 29 \\
\hline 40 & $\mathrm{~B}$ & $5.20 \pm 2.19$ & $\mathrm{~B}$ & $0.5-3.0$ & $9.18 \pm 3.23$ & $16.46 \pm 4.46$ & R. cerealis30 \\
\hline 41 & B & $6.93 \pm 1.26$ & B & $0.5-3.0$ & $9.70 \pm 2.85$ & $25.48 \pm 5.74$ & R. cerealis 31 \\
\hline 42 & $\mathrm{~B}$ & $6.41 \pm 1.34$ & $\mathrm{~B}$ & $0.5-3.0$ & $9.53 \pm 1.60$ & $20.97 \pm 4.95$ & R. cerealis 32 \\
\hline 43 & B & $5.72 \pm 2.24$ & $\mathrm{~B}$ & $0.5-3.0$ & $7.10 \pm 2.07$ & $25.82 \pm 7.89$ & R. cerealis33 \\
\hline 44 & B & $6.41 \pm 1.34$ & $\mathrm{~B}$ & $0.5-3.0$ & $10.22 \pm 1.54$ & $19.06 \pm 4.97$ & R. cerealis34 \\
\hline 45 & $\mathrm{~B}$ & $6.76 \pm 1.31$ & $\mathrm{~B}$ & $0.5-3.0$ & $10.05 \pm 3.65$ & $19.06 \pm 5.78$ & R. cerealis35 \\
\hline 46 & B & $3.98 \pm 1.66$ & $\mathrm{~B}$ & $0.5-3.0$ & $10.58 \pm 2.96$ & $19.87 \pm 6.17$ & R. cerealis36 \\
\hline 47 & $\mathrm{~B}$ & $6.06 \pm 1.26$ & $\mathrm{~B}$ & $0.5-3.0$ & $10.05 \pm 2.38$ & $23.74 \pm 6.04$ & R. cerealis 37 \\
\hline 48 & B & $6.58 \pm 1.34$ & $\mathrm{~B}$ & $0.5-3.0$ & $11.09 \pm 3.32$ & $23.57 \pm 5.51$ & R. cerealis38 \\
\hline 49 & WY & $4.68 \pm 1.45$ & $\mathrm{~B}$ & $1.0-2.0$ & $7.45 \pm 2.16$ & $19.58 \pm 5.09$ & R. fumigata7 \\
\hline 50 & WY & $5.37 \pm 1.82$ & $\mathrm{~A}$ & ---- & $7.80 \pm 1.96$ & $19.41 \pm 4.99$ & R. fumigata 8 \\
\hline 51 & WY & $6.58 \pm 1.34$ & $\mathrm{~B}$ & $1.0-2.5$ & $8.66 \pm 1.26$ & $19.76 \pm 6.57$ & R. fumigate 9 \\
\hline 52 & WY & $4.33 \pm 1.88$ & $\mathrm{~B}$ & $1.0-3.0$ & $7.10 \pm 1.53$ & $24.09 \pm 4.44$ & R. cerealis39 \\
\hline 53 & WY & $5.02 \pm 2.07$ & $\mathrm{~A}$ & ---- & $9.01 \pm 2.16$ & $19.06 \pm 4.57$ & R.fumigata 10 \\
\hline 54 & WY & $5.89 \pm 1.19$ & B & $0.5-1.0$ & $10.40 \pm 1.96$ & $22.88 \pm 3.95$ & R. cerealis 40 \\
\hline 55 & WY & $5.89 \pm 1.19$ & $\mathrm{~B}$ & $0.5-0.1$ & $7.10 \pm 1.19$ & $18.89 \pm 5.68$ & R.fumigate 11 \\
\hline 56 & WY & $5.37 \pm 1.82$ & $\mathrm{~B}$ & $0.5-1.0$ & $7.80 \pm 1.96$ & $18.37 \pm 5.05$ & R.fumigata 12 \\
\hline 57 & WY & $4.33 \pm 1.88$ & $\mathrm{~B}$ & $0.5-1.0$ & $8.35 \pm 1.50$ & $22.65 \pm 3.29$ & R. cerealis 41 \\
\hline 58 & WY & $4.50 \pm 2.07$ & $\mathrm{~B}$ & $0.5-1.0$ & $7.02 \pm 1.18$ & $18.37 \pm 3.32$ & R.fumigata 13 \\
\hline 59 & WY & $4.85 \pm 1.93$ & $\mathrm{~B}$ & $0.5-1.0$ & $7.28 \pm 1.07$ & $19.76 \pm 1.91$ & R. fumigata 14 \\
\hline 60 & WY & $4.68 \pm 2.01$ & B & $0.5-1.0$ & $7.97 \pm 2.68$ & $18.02 \pm 6.62$ & R.fumigata 15 \\
\hline 61 & WY & $5.02 \pm 1.82$ & $\mathrm{~B}$ & $0.5-1.0$ & $8.32 \pm 3.13$ & $17.68 \pm 6.15$ & R.fumigata 16 \\
\hline 62 & WY & $4.87 \pm 1.66$ & $\mathrm{~B}$ & $0.5-1.0$ & $8.49 \pm 3.32$ & $17.16 \pm 5.54$ & R.fumigata 17 \\
\hline 63 & WY & $4.16 \pm 1.64$ & $\mathrm{~A}$ & ---- & $8.80 \pm 2.98$ & $18.72 \pm 3.95$ & R.fumigata 18 \\
\hline 64 & WY & $5.20 \pm 1.96$ & $\mathrm{~A}$ & ---- & $7.80 \pm 2.94$ & $17.68 \pm 6.45$ & R. fumigata 19 \\
\hline 65 & $\mathrm{~B}$ & $4.68 \pm 1.45$ & $\mathrm{~B}$ & $0.5-3.0$ & $8.49 \pm 1.54$ & $22.70 \pm 3.98$ & R. cerealis 42 \\
\hline 66 & $\mathrm{~B}$ & $4.85 \pm 1.66$ & $\mathrm{~B}$ & $0.5-3.0$ & $7.28 \pm 2.44$ & $17.85 \pm 5.27$ & R. cerealis43 \\
\hline 67 & $\mathrm{~B}$ & $4.85 \pm 1.93$ & $\mathrm{~B}$ & $0.5-3.0$ & $7.80 \pm 2.60$ & $17.68 \pm 6.15$ & R. cerealis 44 \\
\hline 68 & $\mathrm{~B}$ & $6.76 \pm 1.31$ & B & $0.5-3.0$ & $9.18 \pm 1.93$ & $18.72 \pm 4.41$ & R. cerealis 45 \\
\hline 69 & $\mathrm{~B}$ & $4.68 \pm 1.75$ & $\mathrm{~B}$ & $0.5-3.0$ & $7.10 \pm 3.02$ & $18.20 \pm 5.89$ & R. cerealis46 \\
\hline 70 & B & $7.62 \pm 0.67$ & $\mathrm{~B}$ & $0.5-3.0$ & $9.36 \pm 1.31$ & $24.78 \pm 7.53$ & R. cerealis 47 \\
\hline 71 & $\mathrm{~B}$ & $6.06 \pm 1.26$ & $\mathrm{~B}$ & $0.5-3.0$ & $8.49 \pm 2.29$ & $18.20 \pm 3.37$ & R. cerealis 48 \\
\hline 72 & WY & $3.64 \pm 1.64$ & $\mathrm{~A}$ & --- & $9.01 \pm 2.75$ & $19.93 \pm 4.57$ & R.fumigate 20 \\
\hline 73 & WY & $6.76 \pm 1.31$ & $\mathrm{~A}$ & --- & $7.80 \pm 1.96$ & $18.37 \pm 5.85$ & R.fumigata21 \\
\hline 74 & WY & $3.98 \pm 1.66$ & $\mathrm{~A}$ & ---- & $9.53 \pm 2.89$ & $16.12 \pm 4.73$ & R.fumigate 22 \\
\hline 75 & WY & $5.02 \pm 1.82$ & $\mathrm{~A}$ & ---- & $7.45 \pm 3.23$ & $17.50 \pm 6.84$ & R.fumigata23 \\
\hline 76 & WY & $5.20 \pm 1.70$ & $\mathrm{~B}$ & $0.3-1.0$ & $7.10 \pm 2.85$ & $18.54 \pm 7.21$ & R.fumigata24 \\
\hline 77 & WY & $6.41 \pm 1.34$ & $\mathrm{~B}$ & $0.3-1.0$ & $9.01 \pm 2.16$ & $19.24 \pm 4.69$ & R.fumigata25 \\
\hline 78 & WY & $6.24 \pm 1.91$ & $\mathrm{~B}$ & $0.3-1.0$ & $8.32 \pm 2.81$ & $19.41 \pm 5.80$ & R.fumigata26 \\
\hline
\end{tabular}

All types were isolated from Qalyubia, except the eighth type (*) was from Sharqia. A: absent, B: brown, LB: light to brown, WY: white to yellow. 
Table 3. Identification of 78 isolates of binucleate Rhizoctonia using the key proposed by Sneh et al. (1991)

\begin{tabular}{|c|c|c|c|}
\hline Species & No. of isolates & Species & Number of isolates \\
\hline$R$. cerealis & 48 & $R$. fumigata & 26 \\
\hline R. calle & 2 & R. species & 2 \\
\hline
\end{tabular}

2. Pathogenicity test in vitro:

Pathogenicity test of 78 binucleate Rhizoctonia isolates showed that all isolates were found to be pathogenic on faba bean seeds in vitro (Table 4). All isolates showed variance degree of pathogenicity, and most of them showed the same degree of pathogenicity as $R$. solani. Four isolates belonged to species $R$. cerealis (Nos. 1, 24, 28 \& 47) were more aggressive on faba bean seeds in vitro as compared by other isolates (Table 4).

Table 4. Pathogenicity test of binucleate Rhizoctoni isolates in vitro

\begin{tabular}{|c|c|c|c|c|c|}
\hline Species & $\begin{array}{c}\text { Disease } \\
\text { index }\end{array}$ & Species & $\begin{array}{l}\text { Disease } \\
\text { index }\end{array}$ & Species & $\begin{array}{c}\text { Disease } \\
\text { index }\end{array}$ \\
\hline R. cerealis 1 & $4.00 \pm 1.00$ & R. cerealis 17 & $2.73 \pm 1.27$ & R. fumigata 10 & $1.93 \pm 0.11$ \\
\hline R. cerealis 2 & $3.00 \pm 1.00$ & R. cerealis 18 & $2.60 \pm 1.03$ & R. cerealis 40 & $1.86 \pm 0.11$ \\
\hline R. cerealis 3 & $1.86 \pm 0.90$ & R. cerealis 19 & $2.66 \pm 0.30$ & R. fumigate 11 & $2.13 \pm 0.23$ \\
\hline R. cerealis 4 & $1.06 \pm 0.11$ & R. cerealis 20 & $3.20 \pm 0.87$ & R. fumigata 12 & $2.66 \pm 0.61$ \\
\hline R. cerealis5 & $1.86 \pm 0.23$ & R. cerealis 21 & $2.40 \pm 0.34$ & $R$. cerealis 41 & $2.13 \pm 0.23$ \\
\hline$R$. species1 & $3.00 \pm 0.72$ & R. cerealis 22 & $2.46 \pm 0.41$ & R. fumigata 13 & $2.06 \pm 0.11$ \\
\hline R. cerealis6 & $1.93 \pm 0.11$ & R. cerealis 23 & $2.40 \pm 0.34$ & R. fumigata 14 & $2.53 \pm 0.50$ \\
\hline R. cerealis 7 & $2.13 \pm 0.23$ & R. cerealis 24 & $3.80 \pm 1.11$ & R. fumigata 15 & $2.46 \pm 0.41$ \\
\hline R. cerealis8 & $2.33 \pm 0.57$ & R. cerealis 25 & $2.66 \pm 0.57$ & R. fumigata 16 & $2.26 \pm 0.64$ \\
\hline R. fumigata 1 & $2.06 \pm 0.30$ & R. cerealis 26 & $2.66 \pm 0.57$ & R. fumigata 17 & $2.60 \pm 0.60$ \\
\hline R. fumigata 2 & $2.26 \pm 0.46$ & R. cerealis 27 & $3.46 \pm 1.50$ & R. fumigata 18 & $1.20 \pm 0.34$ \\
\hline R. fumigata3 & $2.60 \pm 0.87$ & R. cerealis 28 & $3.53 \pm 1.50$ & R. fumigata 19 & $1.86 \pm 0.11$ \\
\hline$R$. species 2 & $1.40 \pm 0.69$ & R. cerealis 29 & $2.80 \pm 1.56$ & R. cerealis 42 & $2.53 \pm 0.11$ \\
\hline R. calle 1 & $2.33 \pm 0.30$ & R. cerealis 30 & $1.60 \pm 0.40$ & R. cerealis 43 & $3.06 \pm 0.90$ \\
\hline R. calle 2 & $2.06 \pm 0.11$ & R. cerealis 31 & $2.20 \pm 0.34$ & R. cerealis 44 & $2.26 \pm 0.46$ \\
\hline R. cerealis 9 & $1.80 \pm 0.72$ & R. cerealis 32 & $3.20 \pm 1.58$ & R. cerealis 45 & $2.06 \pm 0.11$ \\
\hline R. cerealis 10 & $2.20 \pm 0.34$ & R. cerealis 33 & $1.66 \pm 0.30$ & R. cerealis 46 & $2.13 \pm 0.23$ \\
\hline$R$. cerealis 11 & $2.53 \pm 0.50$ & R. cerealis 34 & $1.53 \pm 0.61$ & R. cerealis 47 & $3.53 \pm 0.75$ \\
\hline R. fumigata 4 & $1.20 \pm 0.20$ & R. cerealis 35 & $2.80 \pm 1.92$ & R. cerealis 48 & $2.46 \pm 0.50$ \\
\hline R. fumigata5 & $2.00 \pm 0.00$ & R. cerealis 36 & $3.13 \pm 1.20$ & R. fumigate 20 & $2.26 \pm 0.23$ \\
\hline R. fumigata 6 & $2.00 \pm 0.00$ & R. cerealis 37 & $3.20 \pm 1.63$ & R. fumigata 21 & $2.00 \pm 0.00$ \\
\hline R. cerealis 12 & $1.26 \pm 0.46$ & R. cerealis 38 & $2.73 \pm 0.46$ & R. fumigate 22 & $2.53 \pm 0.50$ \\
\hline R. cerealis 13 & $2.40 \pm 0.69$ & R. fumigata7 & $2.26 \pm 0.46$ & R. fumigata 23 & $2.00 \pm 1.00$ \\
\hline R. cerealis 14 & $2.13 \pm 0.80$ & R. fumigata 8 & $1.86 \pm 0.23$ & R. fumigata 24 & $2.20 \pm 0.20$ \\
\hline R. cerealis 15 & $2.06 \pm 0.11$ & R. fumigate 9 & $2.13 \pm 0.41$ & R. fumigata 25 & $1.46 \pm 0.50$ \\
\hline R. cerealis 16 & $2.93 \pm 0.90$ & R. cerealis 39 & $2.26 \pm 0.46$ & R. fumigata 26 & $1.93 \pm 0.11$ \\
\hline$R$. solani (cont.) & $3.13 \pm 0.61$ & & & & \\
\hline
\end{tabular}




\section{Pathogenicity test under greenhouse condition:}

Four more aggressive isolates (Nos. 1, 24, 28, 47; belonging to $R$. cerealis) in vitro test were chosen to test under greenhouse conditions for pathogenicity and plant growth characters. All isolates showed significantly greater disease incidence on faba bean seedlings, and showed the same effect with polynucleate isolate $R$. solani (AG4-HGI) (Fig. 1). They reduced the percentages of emerged seedlings compared with control plants but their effect was lower than that found by polynucleate $R$. solani (Fig. 2). All isolates (polynucleate isolate and binucleate isolates) significantly decreased both shoot and root weight compared with healthy plants (Figs. 3\&4). On the contrary, no significant effects were recorded to other plant growth characters including stem length and number of leaves/plant (Figs. $5 \& 6)$.

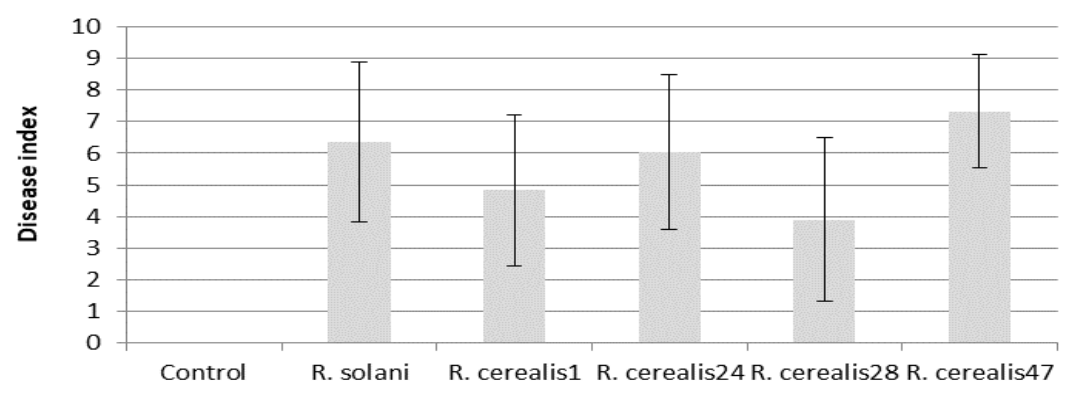

Species

Fig. 1. Pathogenicity test of 4 isolates binucleate Rhizoctonia cerealis under greenhouse condition.

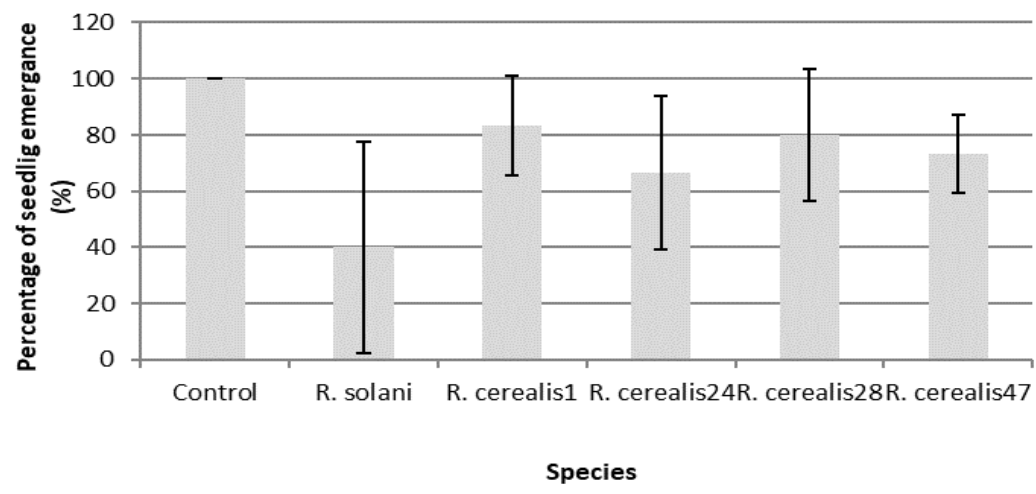

Fig. 2. Effect of 4 isolates binucleate Rhizoctonia cerealis on percentage of faba bean seedling emergence, 30 days after planting. 


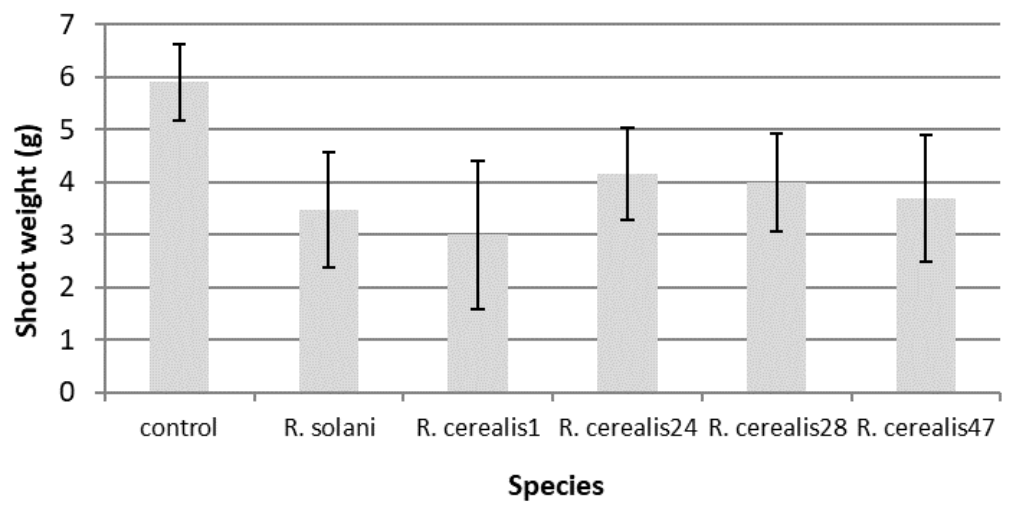

Fig. 3. Effect of 4 isolates binucleate Rhizoctonia cerealis on shoot weight of faba bean plants.

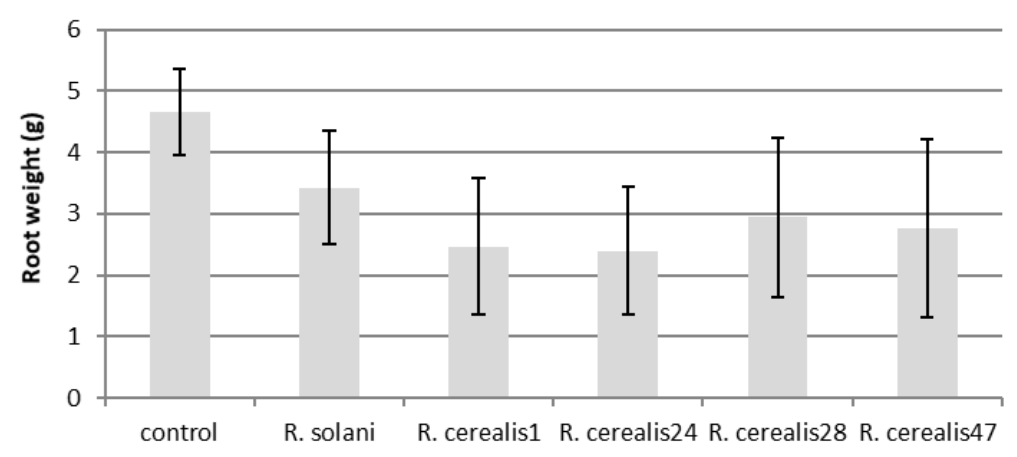

Species

Fig. 4. Effect of 4 isolates binucleate Rhizoctonia cerealis on root weight of faba bean plants.

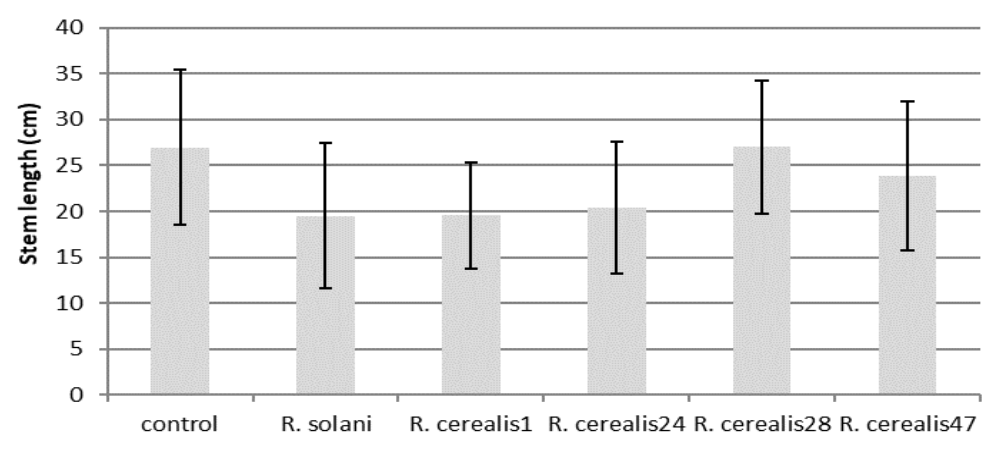

Fig. 5. Effect of 4 isolates binucleate Rhizoctonia cerealis on stem length of faba bean plants. 


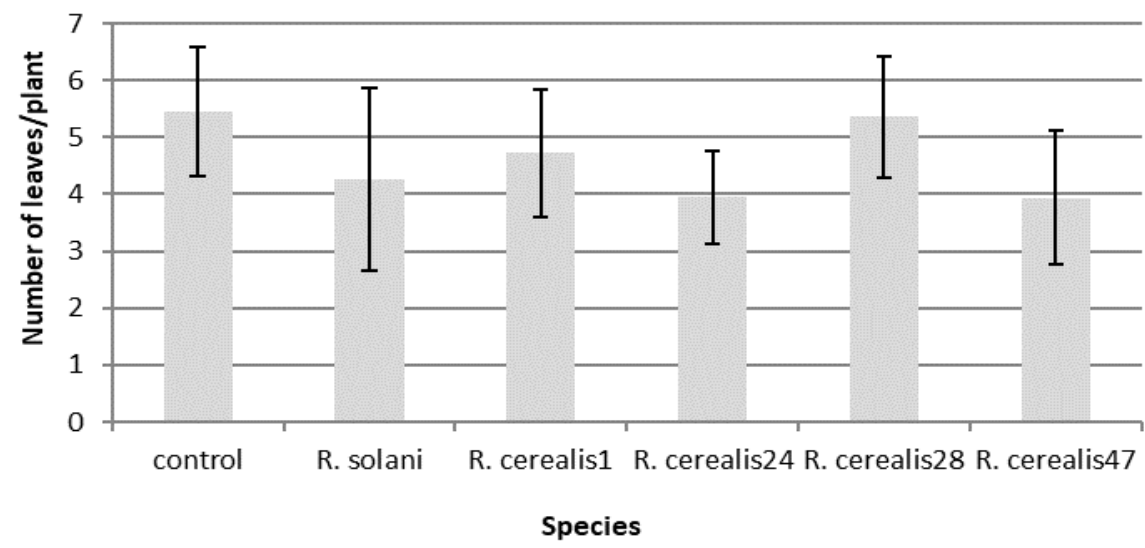

Fig. 6. Effect of 4 isolates binucleate Rhizoctonia cerealis on number of leaves of faba bean plants.

\section{D i s c u s s i on}

Rhizoctonia root rot is an important disease of faba bean plants (Rashad et al., 2012; Chang et al., 2014). Most of researchers focused their studies on the polynucleate $R$. solani as the most common pathogen that has been related with faba bean root rot and with a wide host range (Bolkan and Ribeiro, 1985; Mathew et al., 2012; Chang et al., 2014). This study represented the first attempt to focus on binucleate Rhizoctonia causing root rot of faba bean in Egypt.

The present study aimed to identify and determine the pathogenicity of binucleate Rhizoctoni isolates isolated from faba bean plants showing root rot and stem canker. A total of 104 Rhizoctonia isolates were isolated from roots and basal stems of faba bean plants collected from Qalyubia and Sharqia governorate. Number of Rhizoctonia isolates isolated from plants collected from Qalyubia region was higher than that isolated from Sharqia region and this may be due to the soil type in both regions, soil type in Qalyubia is heavy clay soil and in Sharqia it is light sandy clay soil. Depending on the number of nuclei in cells close to tips of young hyphae (Sneh et al., 1991), Rhizoctonia isolates have been divided into two main groups known as polynucleate and binucleate. Identification of binucleate isolates according to key adopted by Sneh et al. (1991) appeared that two isolates were identified as Rhizoctonia calle, 26 isolates for $R$. fumigatea, 48 isolates to $R$. cerealis and two isolates out of the identification key.

Many researchers showed that binucleate Rhizoctonia spp. are pathogenic to some hosts (Botha et al., 2003; Hyakumachi et al., 2005; Cedeno et al., 2006 Molaei et al., 2014; Ünal et al., 2014). Among of them, Ünal et al. (2014) showed that strains of binucleate Rhizoctonia cerealis, belonged to AG-D isolated from soil samples in wheat production areas from Turkey, were found to be pathogenic on susceptible wheat cultivar. All AG-D isolates showed $41-83 \%$ disease severity values assessed as pathogens. These results were recorded to be the first in the wheat 
field soils in Turkey. Otherwise, there are few studies reporting that binucleate Rhizoctonia are pathogenic on legumes crops (Oniki and Araki, 1981; 1982; Yang et al., 2005).

The present study revealed that all binucleate Rhizoctonia isolates were found to be pathogenic to faba bean germinated seeds in vitro. Isolates were differed in their virulence. Among them, four isolates showed more aggressive belonged to species $R$. cerealis have been selected to test their aggressiveness under greenhouse conditions. Tested binucleate isolates under greenhouse conditions showed greater disease incidence on faba bean root, and showed the same effect as compared with polynucleate $R$. solani (AG4-HGI).

The results of this study are in agreement with Yang et al. (2005), who showed that twenty binucleate Rhizoctonia Chinese isolates belonged to AG-A were pathogenic to pea, soya bean, snap bean and park choy.

According to the key adopted by Sneh et al. (1991), nomenclature of binucleate Rhizoctonia depends mainly on morphological features of fungal growth. Practically it is very difficult to use these parameters for their nomenclature due to great interference of these characters between species. Therefore, it is very important to find out new features of identification of binucleate Rhizoctonia and this will be our aim in the future.

\section{Refer e n c e s}

Anderson, N.A. 1982. The genetics and pathology of Rhizoctonia solani. Annu. Rev. Phytopathol., 20: 329-347.

Araki, T.; Oniki, M. and Ogoshi, A. 1979. Occurrence of tortoise shell-like symptom of potato tubers by binucleate Rhizoctonia. Ann. Phytopathol. Soc. Japan, 45: 530.

Bolkan, H.A. and Ribeiro, W.R.C. 1985. Anastomosis groups and pathogenicity of Rhizoctonia solani isolates from Brazil. Plant Dis., 69: 599-601.

Botha, A.; Denman, S.; Lamprecht, S.C.; Mazzola, M. and Crous, W. 2003. Characterization and pathogenicity of Rhizoctonia isolates associated with black root rot of strawberries in the Western Cape Province, South Africa. Australas. Plant Pathol., 32: 195-201.

Burpee, L. 1980. Rhizoctonia cerealis causes yellow patch of turfgrasses. Plant Dis., 64: $1114-1116$.

Burpee, L. L.; Sanders, P. L.; Cole, H. Jr. and Kim, S. H. 1978. A staining technique for nuclei of Rhizoctonia solani and related fungi. Mycologia, 70: 1281-1283.

Cedeno, L.; Castro, F. and Quintero, K. 2006. Damping-off on alfalfa caused by a binucleate Rhizoctonia in Merida, Venezuela. Fitopatol. Venez., 19(1): 2-4.

Chang, K.F.; Conner, R.L.; Hwang, S.F.; Ahmed, H.U.; McLaren, D.L.; Gossen, B.D. and Turnbull, G.D. 2014. Effects of seed treatments and inoculum density 
of Fusarium avenaceum and Rhizoctonia solani on seedling blight and root rot of faba bean. Can. J. Plant Sci., 94: 693-700.

Cubeta, M. A. and Echandi, E. 1991. Biological control of Rhizoctonia and Pythium damping-off of cucumber: an integrated approach. Biol. Control, 1(3): 227-236.

Eken, C. and Demirci, E. 2003. Identification and pathogenicity of Rhizoctonia solani and binucleate Rhizoctonia anastomosis groups isolated from forage legumes in Erzurum, Turkey. Phytoparasitica, 31(1): 76-80.

Eken, C. and Demirci, E. 2004. Anastomosis groups and pathogenicity of Rhizoctonia solani and binucleate Rhizoctonia isolates from bean in Erzurum, Turkey. J. Plant Pathol., 86: 49-52.

Erper, I.; Karaca, G.H.; Ozkoc, I. and Turkkan, M. 2013. Binucleate Rhizoctonia repens Bernard as a biocontrol agent against damping-off disease of cucumber plants. Eur. J. Plant Sci. Biotechnol., 7(special issue1): 58-61.

Ghahramani, S. 2000. Fundamentals of Probability (2ed). Prentice Hall: New Jersey, $438 \mathrm{p}$.

Herr, L. J. 1995. Biological control of Rhizoctonia solani by binucleate Rhizoctonia spp. and hypovirulent $R$. solani agents. Crop Prot., 14(3): 179-186.

Hyakumachi, M.; Priyatmojo, A.; Kubota, M. and Fukui, H. 2005. New anastomosis groups, AG-T and AG-U, of binucleate Rhizoctonia spp. causing root and stem rot of cut-flower and miniature roses. Phytopathology, 95(7): 784-792.

Mathew, F.M.; Lamppa, R.S.; Chittem, K.; Chang, Y.W.; Botschner, M.; Kinzer, K.; Goswami, R.S. and Markell, S.G. 2012. Characterization and pathogenicity of Rhizoctonia solani isolates affecting Pisum sativum in North Dakota. Plant Dis., 96: 666-672.

Mohamed, M.H.; Gado, E.A.M.; El Deeb, S.H. and Mostafa, M.H. 2014. Effect of nitrate levels as a fertilizer or as a fungal nutrition on the aggressiveness of Rhizoctonia solani on faba bean. Eur. J. Adv. Res. Biol. Life Sci., 2(2): 1-13.

Mohamed, M.H.; Gado, E.A.M.; El-Deeb, S.H. and Mostafa, M.H. 2015a. Phenotypic diversity and molecular identification of the most prevalent anastomosis group of Rhizoctonia solani isolated from diseased faba bean plants. Amer. J. Life Sci., 3(1): 47-55.

Mohamed, M.H.; Gado, E.A.M.; El-Deeb, S.H. and Mostafa, M.H. 2015 b. Obstruction of signal transduction between faba bean and Rhizoctonia solani during pathogenesis and its impact on root rot disease. Int. Sci. Res. J., 1: 99-107.

Molaei, S.; Alaei, H.; Mahmoudi, S.B. and Sedaghati, E. 2014. New anastomosis group AG-G of binucleate Rhizoctonia sp., the causal agent of root rot disease on miniature roses in Iran. Mycologia Iranica, 1(2): 99-106.

Ogoshi, A. 1985. Anastomosis and intraspecific groups of Rhizoctonia solani and binucleate Rhizoctonia. Fitopatol. Bras., 10: 371-390. 
Ogoshi, A.; Oniki, M.; Araki, T. and Ui, T. 1983. Studies on the anastomosis groups of binucleate Rhizoctonia and their perfect states. J. Fac. Agr. Hokkaido Univ., 61: $244-260$.

Oniki, M. and Araki, T. 1981. Occurrence of browning on peanut pods by binucleate Rhizoctonia. Ann. Phytopathol. Soc. Japan, 47: 109.

Oniki, M. and Araki, T. 1982. Fungi isolated from browning of peanut pods (a new disease) and their pathogenicity. Ann. Phytopathol. Soc. Japan, 48: 84.

Parmeter, J.R. and Whitney, H.S. 1970. Taxonomy and nomenclature of the imperfect state. In: Parmeter, J. R. (ed.). Biology and Pathology of Rhizoctonia solani. Univ. of California Press, Berkeley, 225 pp.

Poromarto, S.H.; Nelson, B.D. and Freeman, T.P. 1998. Association of Binucleate Rhizoctonia with soybean and mechanism of biocontrol of Rhizoctonia solani. Phytopathology, 88(10): 1056-67.

Rashad, Y.M.; Abdel-Fattah, G.M.; Hafez, E.E. and El-Haddad, S.A. 2012. Diversity among some Egyptian isolates of Rhizoctonia solani based on anastomosis grouping, molecular identification and virulence on common bean. Afr. J. Microbiol. Res., 6(37): 6661-6667.

Sharma-Poudyal, D.; Paulitz, T.C.; Porter, L.D. and du Toit, L.J. 2015. Characterization and pathogenicity of Rhizoctonia and Rhizoctonia-like spp. from pea crops in the Columbia Basin of Oregon and Washington. Plant Dis., 99: 604-613.

Simonetta, S.; Avidano, L. and Berta, G. 2007. Morphogenetic effects induced by pathogenic and non pathogenic Rhizoctonia solani Kühn strains on tomato roots. Caryologia, 60: 141-145.

Sneh, B.; Burpee, L. and Ogoshi, A. 1991. Identification of Rhizoctonia species. APS Press, St. Paul, Mn. USA, 133 p.

Tuncer, S. and Eken, C. 2013. Anastomosis grouping of Rhizoctonia solani and binucleate Rhizoctonia spp. isolated from pepper in Erzincan, Turkey. Plant Prot. Sci., 49: 127-131.

Ünal, F.; Dolar, F.S.; Yildirim, A.F. and Demirci, E. 2014. Isolation and identification of binucleate Rhizoctonia spp. from wheat field soils in the central Anatolia region, Turkey. Turkish J. Agric. Natural Sci., 2: 1933-1938.

Yang, G.H.; Chen, H.R.; Naito, S.; Ogoshi, A. and Deng, Y.L. 2005. First report of AG-A of binucleate Rhizoctonia in China, pathogenic to soya bean, pea, snap bean and park choy. J. Phytopathol., 153: 333-336.

Yang, Y.; Zhao, C.; Guo, Z. and Wu, X. 2015. Potato stem canker caused by binucleate Rhizoctonia AG-G in China. J. Gen. Plant Pathol., 81: 287-290.

(Received 10/05/2017; in revised form 12/06/2017) 


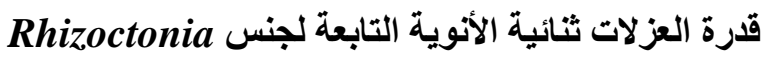 كمسبيات لمرض عفن الجذور في الفول البلدي

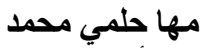 قسم أمر اضي النبات ـ كلية الزر اعة ـ جامعة عين شمس - مصر
}

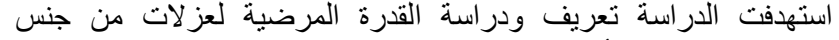
Rhizoctonia

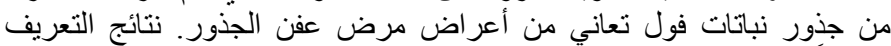

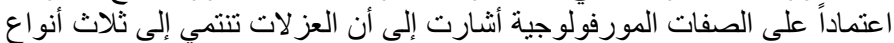

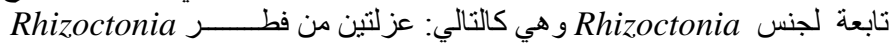
R. R. calle

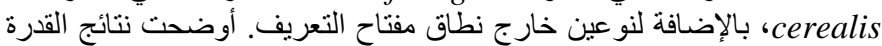

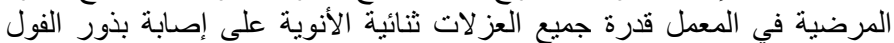

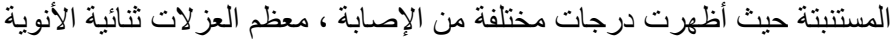

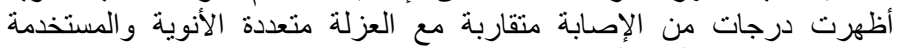

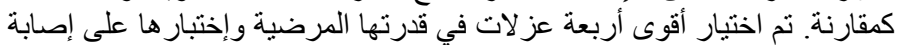

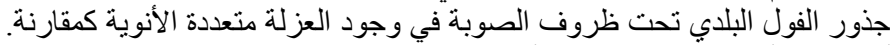

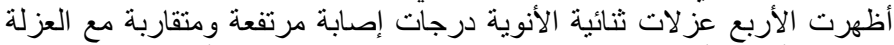

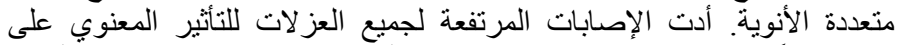

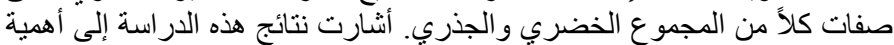
العز لات ثنائية الأنوية كمسبيات لمرض عفن الجذور في الفول البلدي.
\end{abstract}

